IN BRIEF

\title{
A time code for myelin
}

Combinatorial codes are a recurring theme in neural development - a Hox gene code specifies different cell types along the anteroposterior axis of the neural tube, and distinct combinations of transcription factors determine neuronal fate along the dorsoventral axis. Now, in the Journal of Neuroscience, Farhadi et al. describe a new combinatorial code that temporally controls the expression of a single gene. They show that glia use different combinations of regulatory sequences to control the expression of myelin basic protein (MBP) at various stages during and after the onset of myelination.

Farhadi et al. examined the region upstream of the MBP coding sequence, and they identified four regulatory modules - M1-M4 that are conserved between mice and humans. The authors made DNA constructs in which various combinations of regulatory elements were linked to a reporter gene. These reporter constructs were inserted in single copy into the mouse genome using a controlled transgenesis strategy that allowed direct comparisons of both qualitative and quantitative expression phenotypes.

In the central nervous system, Farhadi et al. showed that M1 and M2 upregulated reporter gene expression in oligodendrocytes in the early postnatal period during primary myelination. M3, on the other hand, drove continuous

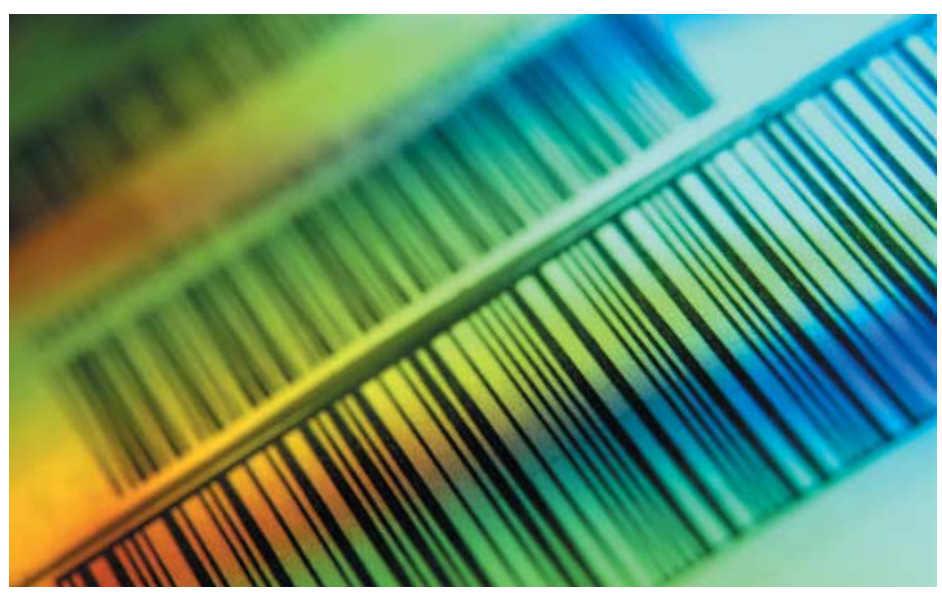

reporter expression throughout primary myelination and adulthood, and the authors proposed that $\mathrm{M} 3$ is required for myelin maintenance. M3 also seems to be required during myelin repair in the CNS after a demyelinating injury. In the peripheral nervous system, both myelinating and remyelinating Schwann cells used elements within M3 and M4 to drive reporter gene expression.

These findings indicate that different phases in myelin development and maintenance are characterized by regulatory elements to control $M B P$ expression. By finding out which factors bind to these elements, it should be possible to identify the upstream signalling pathways that control myelination and remyelination. This could have important implications for myelin repair. Remyelination in the injured adult nervous system produces comparatively thin myelin sheaths, and this tends to limit the degree of functional recovery. If the signalling pathways that promote robust myelination can be identified and harnessed, this problem might be overcome.

Heather Wood

(4) References and links ORIGINAL RESEARCH PAPER Farhadi, H. F. etal. A combinatorial network of evolutionarily conserved myelin basic protein regulatory sequences confers distinct glial-specific remyelination fail in multiple sclerosis? Nature Rev. Neurosci. 3, 705-714 (2002) the use of different combinations of phenotypes. J. Neurosci. 23, 10214-10223 (2003) FURTHER READING Franklin, R. J. Why does 